\title{
In Situ Liquid Crystal Gel as a New Ophthalmic Drug Delivery System for Pilocarpine Nitrate: Improving Preocular Retention and Ocular Bioavailability
}

\section{Wenqing Wu}

Anhui University of Traditional Chinese Medicine

\section{Wenxuan Cao}

Anhui University of Traditional Chinese Medicine

Ye Cai

Anhui University of Traditional Chinese Medicine

Menghe Zhang

Anhui University of Traditional Chinese Medicine

Tiantian Chen

Anhui University of Traditional Chinese Medicine

Nannan Zhang

Anhui University of Traditional Chinese Medicine

Mengzhen Wang

Anhui University of Traditional Chinese Medicine

Lin Chu

Anhui University of Traditional Chinese Medicine

xiaoqin Chu ( $D$ XiaoqinChu113@163.com)

Anhui University of Traditional Chinese Medicine

\section{Research Article}

Keywords: Pilocarpine nitrate, Liquid crystal gel, Ophthalmic administration, Corneal penetration, Glaucoma

Posted Date: July 26th, 2021

DOI: https://doi.org/10.21203/rs.3.rs-644389/v1

License: (c) (1) This work is licensed under a Creative Commons Attribution 4.0 International License.

Read Full License 

system for pilocarpine nitrate: improving preocular retention and

\section{ocular bioavailability}

Running title: Application of in-situ liquid crystal gel in ocular drug delivery

Wenqing $\mathrm{Wu}^{\mathrm{a}}$, Wenxuan $\mathrm{Cao}^{\mathrm{a}}$, Ye Cai ${ }^{\mathrm{a}}$, Menghe Zhang ${ }^{\mathrm{a}}$, Tiantian Chen ${ }^{\mathrm{a}}$, Nannan Zhang ${ }^{\mathrm{a}}$, Mengzhen Wang ${ }^{\mathrm{a}}$, Lin $\mathrm{Chu}^{\mathrm{a}}$, Xiaoqin $\mathrm{Chu}^{\mathrm{a}, \mathrm{b}^{*}}$

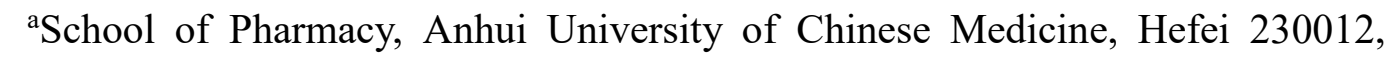
China

bInstitute of Pharmaceutics, Anhui Academy of Chinese Medicine, Hefei 230012, China

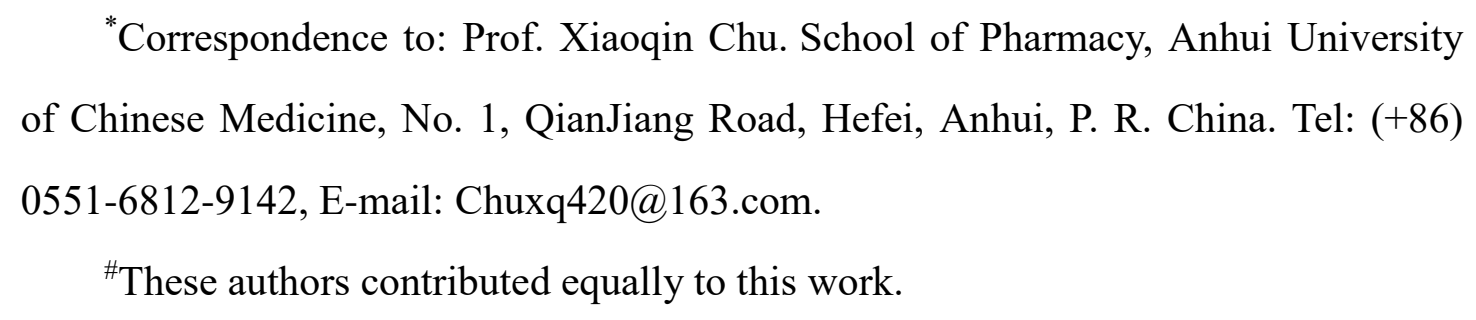

${ }^{*}$ Correspondence to: Prof. Xiaoqin Chu. School of Pharmacy, Anhui University of Chinese Medicine, No. 1, QianJiang Road, Hefei, Anhui, P. R. China. Tel: (+86) 0551-6812-9142, E-mail: Chuxq420@163.com.

${ }^{\#}$ These authors contributed equally to this work.

(1)

19

0

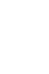

23




\section{In situ liquid crystal gel as a new ophthalmic drug delivery system} for pilocarpine nitrate: improving preocular retention and ocular

\section{bioavailability}

Abstract: The purpose of this article is to develop an in-situ liquid crystal gel that can be used as a novel ocular delivery system for pilocarpine nitrate (PN). The phytantriol (PT) -based in situ liquid crystal gels were prepared by a vortex method using PT, PEG400, Triglyceride (TAG) and water (in the ratio of 61.15:30:3.85:5, w/w). Firstly, the internal structure of the PN-loaded liquid crystal gel was characterized by polarizing microscope (PLM), small-angle X-ray scattering (SAXS), differential scanning calorimetry (DSC) and rheology. In vitro drug release behavior and ex vivo corneal permeation were investigated. Finally, eye irritation test, preocular residence time evaluation,were studied in vivo and compared with eye drops.Based on various characterization techniques, it is proved that the internal structure of the gel is a hexagonal phase.In vitro release results identified that PN could be released continuously from HII gel over a period of $24 \mathrm{~h}$. The in vitro obvious permeability coefficient of HII gel was 3.19-fold $(\mathrm{P}<0.01)$ higher than that of the eye drops. Compared with eye drops,the HII gel had good bioadhesion and displayed longer residence time on the eyeballs surface using fluorescent labeling technology.In addition, through Corneal hydration level and eye irritation test ., it is conjectured that $\mathrm{H}_{\text {II }}$ gel will not cause eye irritation. In short, the formulation has the advantages of high efficiency, slow release and non-toxicity, and will become a promising pharmaceutical strategy to improve the efficacy of glaucoma.

Key words: Pilocarpine nitrate, Liquid crystal gel, Ophthalmic administration, Corneal penetration, Glaucoma 
60

61<smiles>CCC1C(=O)OCC1Cc1ccn(C)c1</smiles>

62

63

$\mathrm{PN}+\mathrm{TAG}+\mathrm{H}_{2} \mathrm{O}$

$64 \quad$ In situ gel
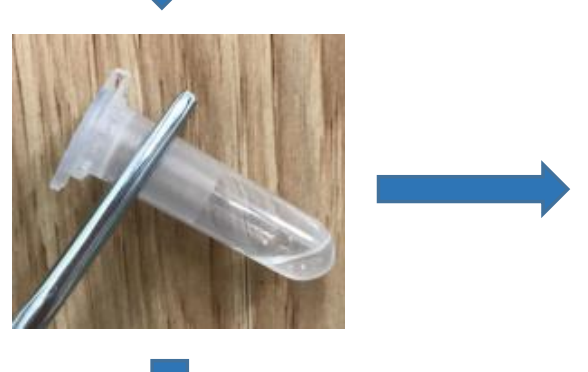

65

66
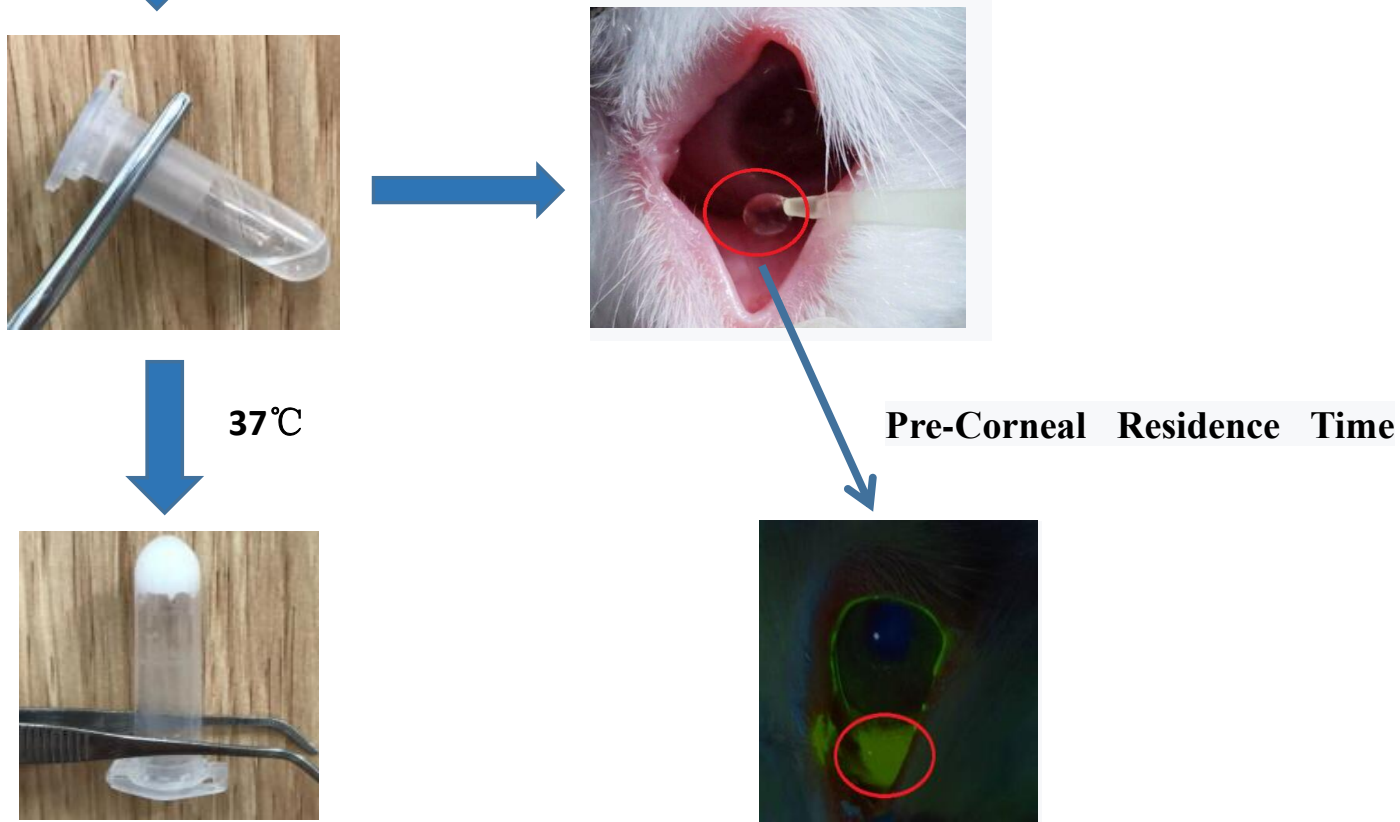

67

Gel

68

69

Characterization
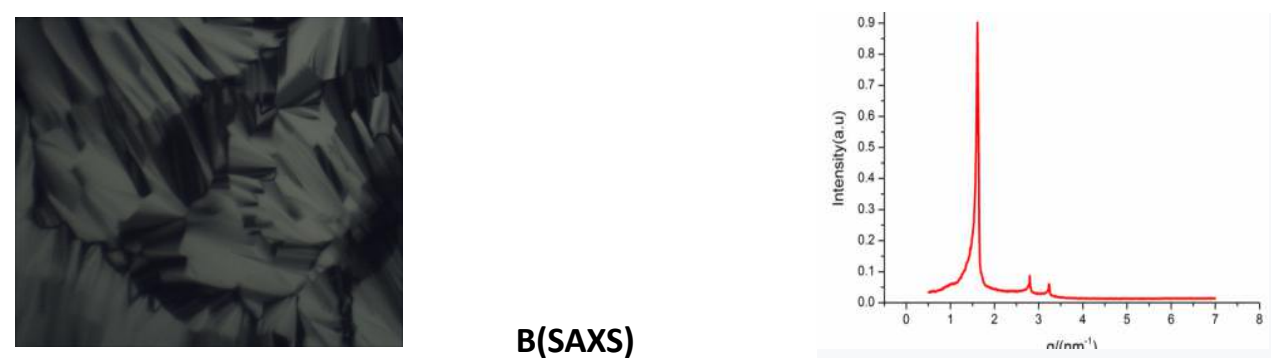

B(SAXS)
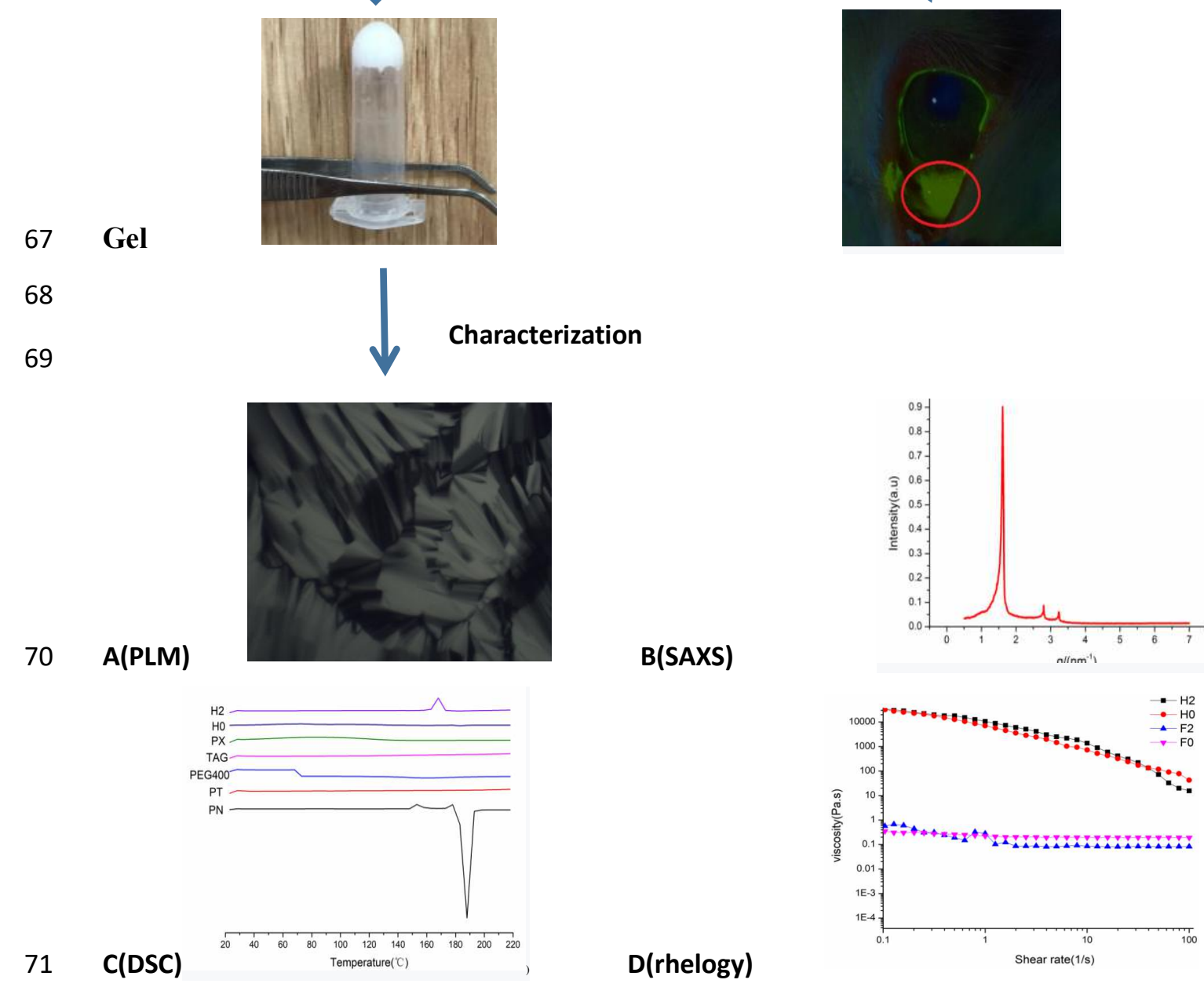


\section{1、Introduction}

Topical ophthalmic administration is the main method of ophthalmic treatment. However, due to the high sensitivity of the eye and unique physiological barriers (including corneal and conjunctival barriers, blood-water barriers and blood-retinal barriers), the drug has low bioavailability and poor efficacy. Commonly used ocular dosage forms are topical eye drops and ophthalmic ointments. However, most of the liquid is discharged from the lacrimal duct after the eye drops are administered, and may cause systemic toxicity after being absorbed through the nasopharynx. Due to its greasy properties and blurred vision, eye ointment has poor patient compliance when applied to eye ointment ${ }^{[1]}$.

Over these years, a growing number of innovative drug delivery systems have been applied to the eyes. Such as liposome nanoparticles and microemulsion, to extend the retention time of the eyes, thus reducing the frequency of medication and increasing bioavailability. However, their drug delivery potential in ophthalmology is limited by the rapid clearance of the anterior cornea due to the same rapid clearance as the water-soluble eye drops. Therefore, considering the unique physiological structure of the eye, effective ocular drug delivery still faces many challenges and needs to find a more effective ocular delivery system.

In recent years, in-situ gel has become a new type of sustained release system ${ }^{[2,3]}$. It is a precursor for administration and then transforms into a gel at the drug delivery site. Compared with other ophthalmic preparations, the precursor preparation of liquid crystal gel has a longer residence time in the eye and good curative effect. In addition, in situ gel is in the form of ordinary solution, similar to eye drops, so the drug is simple and good compliance ${ }^{[4-8]}$. Conversion from solution to gel is usually caused by some physiological or external stimuli, such as variances in $\mathrm{pH}$ value, ionic strength or temperature between the formulation outside the body and the internal tissues ${ }^{[9]}$. When the formulation is exposed to artificial tears, the precursor solution is transparent and can spontaneously become a gel. Since the triggering factor of this phase change is due to the existence of the water environment, it can also be realized 
directly in the physiological fluid. Compared with other methods, such as ultraviolet light and temperature, this method can cause phase transition ${ }^{[10]}$ more mild. According to the literature, it is found that many kinds of in-situ gels have been used in eye administration to improve ocular bioavailability like use of temperature-dependent in-situ gelation polymer (Poloxamer) ${ }^{[4-8]}$, pH-dependent in-situ gelling polymers (Carbopol and Hypromellose) $)^{[11-13]}$, and ionic strength-dependent in-situ gelling polymer (Gellan gum). However, there are few reports on in situ liquid crystal gel for ocular drug delivery.

An amphiphilic substance containing a hydrophilic head group and a hydrophobic hydrocarbon chain domain self-assembles after adding water to form a long-range ordered structure called a lyotropic liquid crystal phase ${ }^{[8]}$. For example, lamellar phase $(\mathrm{La})$, reverse bicontinuous cubic phase $\left(\mathrm{Q}_{\mathrm{II}}\right)$ and reverse bicontinuous hexagonal phase $\left(\mathrm{H}_{\mathrm{II}}\right)$ have received more and more attention due to their unique internal frame and broad drug capacity. Among them, the hexagonal liquid crystal as a drug carrier has attracted widespread interests due to its good stability, potential drug slow-release ability ${ }^{[14]}$. Phytotriol(PT) (shown in Fig.1) are generally considered safe drug matrix and amphiphilic lipids with good mucosal adhesion and biocompatibility, which was often utilized to form liquid crystals. The lyotropic liquid crystal delivery system has many advantages, such as simple preparation, easy to use, reduced dose, and sustained release effects, PT-based liquid crystal gels have been used in the treatment of rheumatoid arthritis and postoperative analgesia ${ }^{[15]}$. However, there are few studies on PT in ocular delivery systems.

Glaucoma is one of the major public health problems in the world. This is a chronic progressive eye disease caused by the apoptosis of retinal ganglion cells and subsequent degeneration of nerve tissue. According to the survey, more than 70 million people in the world suffer from glaucoma, and about $10 \%$ of them are blind, which makes it the main cause of irreversible blindness in the world $2020^{[16]}$. However, this number may rise to 11.1 million by 2020 . Pilocarpine nitrate is a drug that directly acts on the parasympathetic nerve. It can directly stimulate muscarinic receptor $\mathrm{M}$ of iris and ciliary body, cause the contraction of iris and ciliary body, open 
the anterior chamber angle, promote the outflow of aqueous humor through trabecular meshwork structure, and produce the effect of lowering intraocular pressure ${ }^{[17]}$. It has been used in the treatment of chronic open Angle glaucoma and acute closed Angle glaucoma for more than 100 years ${ }^{[18]}$. At the same time, PN has been used the longest as a mainstay drug for glaucoma therapy, and is one of the least-expensive and the most readily available medications ${ }^{[19]}$. Applied in the eye, $\mathrm{PN}$ can penetrate the eye wall with miosis beginning in 15-30 min which last up to 4-8 $\mathrm{h}^{[20]}$. Due to poor corneal permeability, short anterior corneal retention time and poor anterior corneal tear flushing, PN eye drops need to be administered frequently, generally 3-6 times a day ${ }^{[21]}$. Therefore, the bioavailability is extremely low (less than $5 \%$ or even less than $1 \%)$. At the same time, frequent daily administration can cause a series of side effects, such as pupil contraction and myopia, and even a series of gastrointestinal reactions.In addition, our previous research has developed a liquid crystal gel. Based on this, we made the gel into a precursor to improve compliance ${ }^{[22]}$. 
1. Materials and methods

1.1 Materials

PT was acquired from Shanghai Aladdin Biotechnology Co., Ltd. (Shanghai, China).PN was bought from Shanghai TargetMol Biotechnology Co., Ltd. (Shanghai, China). Triglyceride (TAG) was bought from Sigma-Aldrich Trading Co., Ltd. (Shanghai, China). PEG400 was bought Beijing Solarbio \& Technology Co., Ltd Ltd. (Shanghai, China).

The weight of adult New Zealand white rabbit was in the range of 2.5-3.0 kg, which is supplied by the Animal Experimental Center of Anhui University of Chinese Medicine (Hefei, China). All animal experiments were performed in accordance with the guidelines approved by the ethics committee of Anhui University of Chinese medicine (Hefei, China)

\subsection{Prescription Screening}

\subsubsection{Preparation}

According to the literature ${ }^{[24]}$ and our former research (Fig.2), the formulating method was as follows. Firstly, appropriate amount of PT and TAG were mixed and heated to $60 \pm 0.5^{\circ} \mathrm{C}$ to prepare oil phase. Secondly, PN was placed in water at $60 \pm$ $0.5{ }^{\circ} \mathrm{C}$ to prepare aqueous phase (PEG400 and water). Finally, the water phase in the same temperature was added to the oil phase rapidly, and the mixture was immediately rotated for 5 minutes to obtain a liquid crystal gel precursor.

Then, PT-TAG (oil phase) and PEG400-water (water phase) systems were used to gradually change the weight ratio of the prescription from $10 \%: 90 \%$ to $90 \%: 10 \%$. The prescription was screened by the above-mentioned preparation method, and four blank formulas were prepared, namely P 0, P 1, P 2 and P 3. The optimal proportion of stable self-assembled liquid crystal system was determined. Considering the solubility of the drug and the particularity of ophthalmic administration, three preparations were made with drug contents of $0 \%(\mathrm{~F} 0), 0.5 \%(\mathrm{~F} 1), 1 \%(\mathrm{~F} 2)$ and $1.5 \%(\mathrm{~F} 3)$, respectively. After $72 \mathrm{~h}$ of stabilization, the appearance of the preparations was observed. 


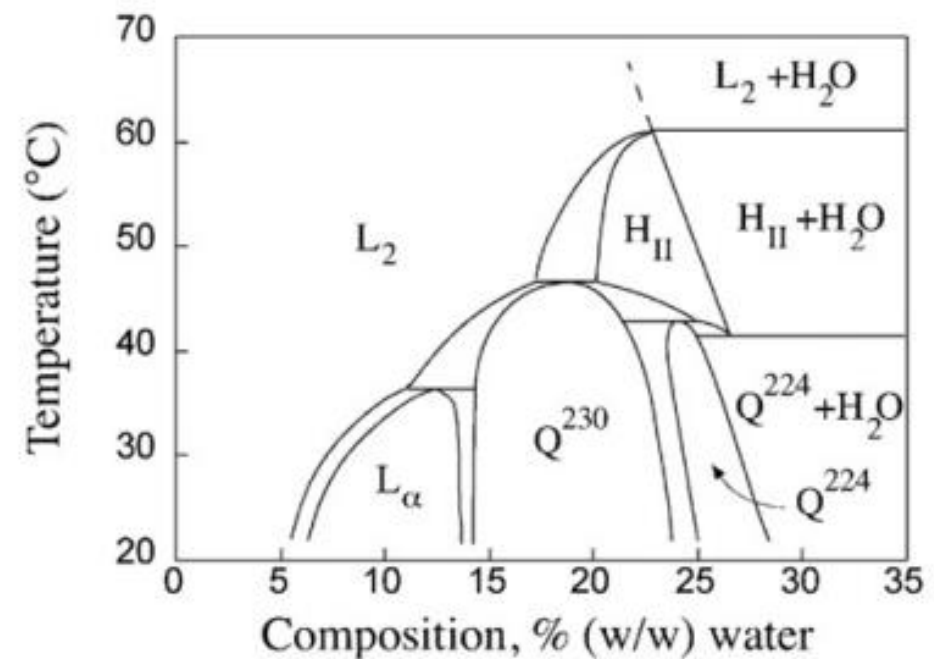

186

187

188

189

190

191

192

193

194

195

196

197

198

199

200

201

202

203

204

205

Fig.2 Temperature composition phase diasgram of the phytantriol/water binary system $^{[25]}$.

\subsubsection{Phase Conversion}

2.2.2.1 The Minimum Volume $\left(\mathrm{V}_{\mathrm{m}}\right)$ and Time $\left(\mathrm{T}_{\mathrm{g}}\right)$ for Phase Conversion

In the rotor method ${ }^{[26]}, 200 \mathrm{mg}$ of the formulation is accurately weighed and added to a tube with a rotor $(10 \mathrm{~mm} \times 6 \mathrm{~mm})$. The test tube was immersed in a water bath at $37{ }^{\circ} \mathrm{Cwith}$ a stirring speed of $30 \mathrm{rpm}$ for 5 minutes. Then, $10 \mu \mathrm{L}$ of artificial tears were added to the centrifuge tube every 1 minute until the rotor was completely stopped due to gelation. The time when the rotor stops moving due to gelation is determined as $\mathrm{Tg}$, and the total volume of water added at this time is determined as $\mathrm{Vm}$.

\subsubsection{Determination of the gelling capacity}

In order to simulate the gelation ability in the human environment, the precursors were placed in a centrifuge tube containing $2 \mathrm{~mL}$ fresh simulated tears $(\mathrm{pH} 7.4$, $37 \pm 1^{\circ} \mathrm{C}$ ) to determine the gelling ability, the time taken for its gelling formation then dissolution of the gels were visually observed and the gelling capacity were evaluated ${ }^{[27]}$ as follows: 


\begin{tabular}{cc}
\hline$(-)$ & No gelation \\
$(+)$ & The gel formed after few minutes and dissolved rapidly \\
$(++)$ & Immediate gelation and remains for few hours \\
$(+++)$ & Immediate stiff gelation which remains for extended period of time \\
\hline
\end{tabular}

2.2.3 Determination of the $\mathrm{pH}$ and osmotic pressure

$\mathrm{pH}$ was measured at $25{ }^{\circ} \mathrm{C}$ by a Model PHS-3C pH-meter (Shanghai Precision Scientific Instrument Co., Ltd). The freezing point method of a Model FM-9X Osmometer (Shanghai Precision Scientific Instrument Co., Ltd) was used to evaluate

211 the osmotic pressure.

\subsection{Characterization of structure}

\subsubsection{PLM}

Polarized light microscope observes the internal structure of the precursor and the liquid crystal gels (CK-500, Shanghai Caikon Optical Instrument Co., Ltd. Shanghai, China ${ }^{[28]}$. Observed by PLM, the isotropic liquid and $\mathrm{Q}_{\mathrm{II}}$ have no birefringence, and the background is dark. The characteristic of La and $\mathrm{H}_{\text {II }}$ is a birefringent structure, that is, $\mathrm{H}_{\text {II }}$ has a fan-shaped structure. Greasy streak texture or Maltese cross shape can be observed in the La phase ${ }^{[29]}$.

\subsubsection{SAXS}

Further structure analysis and phase identification of LC gels were carried out by SAXS measurement (Anton Paar,Graz, Austria) at room temperature. The specimen were tested at $40 \mathrm{kV}$ and $50 \mathrm{~mA}$ for $10 \mathrm{~min}$ using an X-ray source $(\mathrm{Cu}$ Ka radiation,

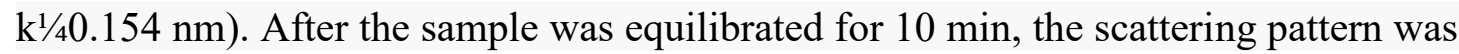
collected, and the scattering intensity was plotted against the q value, which enabled the identification of the peak position. The type of liquid crystal was determined by the peak scattering vector ratio ${ }^{[30]}$.

The relevant parameters of the liquid crystal structure are calculated by the following formulas ${ }^{[31]}$ :

$$
q=(4 \pi \sin \theta) / \lambda
$$


Where $\theta$ is the scattering angle, $\mathrm{q}$ is the position of the first peak, $\mathrm{k}$ is the wavelength of $0.154 \mathrm{~nm}, \mathrm{~d}$ is the distance between the reflecting interplanar space of the liquid crystalline phase, $\alpha$ is the LP which indicates the size of aqueous channels in the LC internal structure, and $\mathrm{h}, \mathrm{k}$, and 1 are the Miller indices and have no dimension.

DSC is used to evaluate temperature and enthalpies of phase transitions during

A DHR-2 rheometer (TA Instruments, New Castle, DE) was used to evaluate the rheological characteristics of LC gels and incorporate a cone-plate sensor with a cone angle of $1^{\circ}$ and a diameter of $20 \mathrm{~mm}$. In the rheological test, the flow rate of the liquid crystal gel was studied by controlling the shear rate from 1 to $100 \mathrm{~s}^{-1}$ during the experiment, and the temperature was maintained at $37 \pm 2{ }^{\circ} \mathrm{C}$. Meanwhile, frequency scanning was used to further evaluate the rheological behavior of the preparation. Finally, a temperature scan was used to study the viscosity change with increasing temperature, and the temperature setting range was $25 \sim 45{ }^{\circ} \mathrm{C}$.

\subsection{Evaluation of in vitro properties}

3.3.1In vitro release study 
The in vitro release of PN from liquid crystal was studied by dynamic dialysis ${ }^{[33]}$. In short, $50 \mathrm{mg}$ fresh PN liquid crystal gels were transferred into dialysis bags (MWCO 8KD-14KD Aladdin,USA) to meet the dialytic capacity of the drug. $150 \mu$

L of eye drops were used as a control. Both eye drops and PN liquid crystal gel contain $1.5 \mathrm{mg}$ of PN. Then, the dialysis bag was immersed in $12 \mathrm{~mL}$ of artificial tears at $37 \pm 1{ }^{\circ} \mathrm{Cunder}$ agitation at $100 \mathrm{rpm}$.

At predetermined time intervals, 1 mlsamples were taken at $0.17,0.5,1.00,1.50$, $2.00,4.00,8.00,12.00,24$ h. Simultaneously, the STF of the same volume was replaced. The samples were filtered by $0.45 \mu \mathrm{m}$ microporous membrane, and the drug content was determined by HPLC. The cumulative release percentage (\%) was plotted against the time $(\mathrm{T}, \mathrm{h})$. The cumulative drug release (Qn, $\mu \mathrm{g} / \mathrm{mL}$ ) was calculated by the following formula:

$$
Q_{n}=c_{n} \times v_{0}+\sum_{i=1}^{n-1} c_{i} \times v_{i}
$$

$C_{n}$ represents the concentration of $\mathrm{PN}$ at each sampling time. $V_{0}$ stand for the volumes of the dissolution medium. $V i$ represents sample volume. $C i$ represents the PN concentration of the ith sample.

The dynamics and mechanisms of PN release in the gel were evaluated by fitting the zero order, first order, Higuchi equation and Ritger-Peppas model to evaluate the dynamic model with the highest correlation coefficient. The release kinetics and mechanism of liquid crystal gel were fitted by the following formula:

Zero order model equation: $y=k 1 t+a l$

First order model equation: $\operatorname{In}(100-y)=k_{2} t+a_{2}$

Higuchi equation: $y=k 3 t^{0.5}+a 3$

Ritger peppas model equation: $y=k 4 t^{n}$

In the above formulas, $y$ is the cumulative release percentage. $t$ is the sampling time; $K_{1}, K_{2}, K_{3}$ and $K_{4}$ are the release rate constants of the equation; $a_{1}, a_{2}$ and $a_{3}$ are constants; and $n$ is the release index describing the release mechanism.

3.3.2 In vitro corneal permeability study 
Remove the eyeballs of New Zealand rabbits that have been derived, and immediately remove the cornea into glutathione bicarbonate ringer(GBR) buffer. The modified Franz diffusion cell was used for in vitro corneal penetration studies, and the effective diffusion area was $0.5024 \mathrm{~cm}^{2}$. Then, the corneas were fixed between the receptor and the donor compartments with the epithelial side facing the donor chamber. Each formulation $(50 \mathrm{mg})$ with concentration of $1.50 \mathrm{mg}$ PN was transferred to the donor compartment, and then the receptor chamber was introduced into $5 \mathrm{~mL}$ GBR solution pre-adjusted to a temperature of $37{ }^{\circ} \mathrm{C}$. Simultaneously, $100 \mu \mathrm{L}$ PN eye drops (containing $1.50 \mathrm{mg} \mathrm{PN}$ )was used as the control group. The samples $(0.4$ $\mathrm{mL})$ were collected at specified intervals $(30,60,90,120,180,240,300$ and $360 \mathrm{~min})$ and replaced with fresh GBR of the same volume ${ }^{[34]}$. The corneal penetration test for each formulation was repeated three times. The samples were filtered by $0.22 \mu \mathrm{m}$ microporous membrane. The PN concentration of samples was determined by HPLC as described above with correction for the volume replacement. The amount of PN that permeated the corneal epithelium was plotted versus time and the slope of the linear portion of the graph was calculated. The steady-state flux $\left[\mathrm{Jss}, \mu \mathrm{g} /\left(\mathrm{cm}^{2} \cdot \mathrm{s}\right)\right]$ and apparent permeability coefficient (Papp, $\mathrm{cm} / \mathrm{s}$ ) were determined as follows:

$$
\begin{gathered}
J_{s s}=C_{0} * P_{a p p} \\
P_{a p p}=\frac{\Delta Q}{\Delta t \cdot C_{0} \cdot A \cdot 60}
\end{gathered}
$$

$\Delta Q / \Delta t$ represents the slope of the linear part of the drug content $(\mathrm{Qn} / \mu \mathrm{g})$ in the receiving pool versus time; $C_{0}$ represents the initial concentration of the drug in the donor cell $(\mathrm{g} / \mathrm{mL}) \cdot A$ represents the corneal surface area $\left(0.5024 \mathrm{~cm}^{2}\right.$ in this study), and 60 is the factor used to convert minutes into seconds.

\subsubsection{Corneal hydration level}

In order to evaluate the irritation of the preparation on the cornea, the corneal hydration level (HL) was measured after in vitro corneal permeability test. At the end 
315 of the in vitro penetration study, each cornea was rinsed with normal saline to remove 316 the residual preparation on the corneal surface, and then weighed. Then, after drying 317 at $70 \pm 0.5{ }^{\circ} \mathrm{C}$ for $12 \mathrm{~h}$, the sample were weighed. In general, The HL value of 318 healthy cornea was $76 \sim 80 \%$, corneal hydration value of more than $83 \%$ indicates 319 some degree of corneal injury ${ }^{[35]}$. The HL\% value was calculated using the following 320 equation $^{[36]}$ :

$$
H L \%=\frac{W_{t}-W_{d}}{W_{t}} \times 100
$$

322

3.4 In vivo performance evaluation

\subsubsection{In vivo eye irritation studies}

The potential ocular irritancy and/or damaging effects of the formulations components and eye drops were evaluated according to a modified Draize test on male New Zealand albino rabbits $(n=6)^{[37]}$. All rabbits were randomly divided into two groups with three rabbits in each group, In the single dose trial, all samples were dripped into the inferior conjunctival sac of each rabbit's right eyes, the left eyes of the contralateral side were treated with saline. After administration, gently close the eyelids for about 10 seconds to avoid loss of preparation. Observe eye reactions (redness, swelling, conjunctival edema, iris and corneal damage, etc.) at 5, 15, 30 minutes and $1,2,3,5,9,12$, and 24 hours after administration. In the multiple administration test, the eye tissue reaction was observed after 1, 2, 3, 4, 5, 6, 7 days according to the method of single administration. Then, according to the evaluation criteria in Table 1, the irritation of the samples to the eyes were judged.

Table 1. Classification of eye irritation

\begin{tabular}{cc}
\hline Score & Stimulus level \\
\hline $0-3$ & Nonirritant \\
$4-8$ & Slight irritation \\
$9-12$ & Medium irritant \\
$13-16$ & Severe irritation \\
\hline
\end{tabular}




\subsubsection{Pre-Corneal Residence Time Analysis}

In this experiment, sodium fluorescein-loaded formulations (i.e. LC gels and solution) were prepared to evaluate their in vivo preocular residence according to a previously reported protocol. Generally, $1 \%$ sodium fluorescein was used to replace PN in the preparation, and then the preparation containing sodium fluorescein was prepared by the same method as the liquid crystal gel precursor.Then, $50 \mathrm{mg}$ of the precursor preparation and $50 \mu \mathrm{L}$ of fluorescein sodium solution were dropped on the lower dome of the rabbit eyes, and finally, the fluorescence intensity was monitored with a fluorescent lamp and excited with blue-light-activated fluorescent lamp.

3 Results

\subsection{Prescription screening}

\subsubsection{Screening and optimization of prescription}

In 2003, Barauskas and Landh reported the phase diagram of PYT/water binary system $^{[38]}$. In addition, we fully considered the solubility of PN and the basis of our previous work in selecting the formula ratio, and prepared four blank liquid crystal gel precursors(P0, P1 ,P2, P3). The above samples need to be balanced at room temperature for $72 \mathrm{~h}$, and then the appearance of the samples can be observed by naked eyes under good light conditions. As shown in Table 2. P1 (PT: PEG: TAG: water $=61.15: 30: 3.85: 5$ ) has better fluidity and better transparent appearance than other preparations. Therefore, considering the solubility of PN, the preparation contains $0.5 \%(\mathrm{~F} 1), 1 \%(\mathrm{~F} 2), 1.5 \%$ (F3) PN drug-loaded preparation.

Table 2 Formulation and drug loading screening of in situ hexagonal liquid crystal

$\begin{array}{llllll}\text { Formulations } & \mathrm{PT}(\mathrm{wt} \%) & \mathrm{PEG} 400(\mathrm{wt} & \mathrm{TAG}(\mathrm{wt} \%) & \text { Water(wt\%) } & \mathrm{PN}(\mathrm{wt} \%)\end{array}$

$\%)$

P0

61.15

32.50

3.85

2.50

0

Milky 


\begin{tabular}{|c|c|c|c|c|c|c|}
\hline $\mathrm{P} 1$ & 61.15 & 30.0 & 3.85 & 5.00 & 0 & $\begin{array}{c}\text { Clarify, } \\
\text { Good } \\
\text { liquidity }\end{array}$ \\
\hline $\mathrm{P} 2$ & 61.15 & 27.5 & 3.85 & 7.50 & 0 & $\begin{array}{c}\text { Milky, Poor } \\
\text { liquidity }\end{array}$ \\
\hline P3 & 61.15 & 25.00 & 3.85 & 10.00 & 0 & $\begin{array}{c}\text { Milky, Poor } \\
\text { liquidity }\end{array}$ \\
\hline F0 & 61.15 & 30.00 & 3.85 & 5.00 & 0 & $\begin{array}{c}\text { Clarify, } \\
\text { Good } \\
\text { liquidity }\end{array}$ \\
\hline $\mathrm{F} 1$ & 61.15 & 30.00 & 3.85 & 5.00 & 0.50 & $\begin{array}{c}\text { Clarify, } \\
\text { Good } \\
\text { liquidity }\end{array}$ \\
\hline F2 & 61.15 & 30.00 & 3.85 & 5.00 & 1.00 & $\begin{array}{l}\text { Clarify, } \\
\text { Good } \\
\text { liquidity }\end{array}$ \\
\hline F3 & 61.15 & 30.0 & 3.85 & 5.00 & 1.50 & $\begin{array}{c}\text { Milky, Poor } \\
\text { liquidity }\end{array}$ \\
\hline
\end{tabular}

366

367 3.1.2 The Minimum Volume (Vm) and Time (Tg) for Phase Conversion

368 The appearance before and after phase inversion was shown in Fig.3. When the

369 liquid crystal precursor was exposed to artificial tears, it undergoes a phase change

370 due to the presence of an aqueous environment, and the precursor solution gradually

371 becomes a gel state. Experimental results showed that $80 \mu \mathrm{L}$ of artificial tears need to

372 be added to $200 \mathrm{mg}$ of the precursor preparation to make the phase change into a gel

373 state, and the time required is about 1.5 to 2 secon 

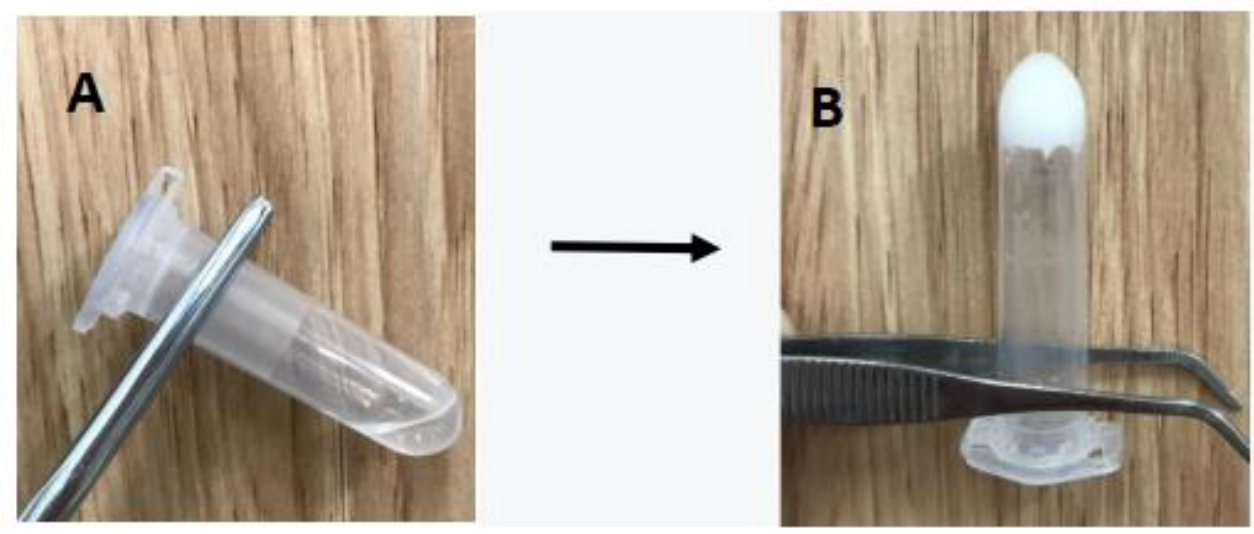

Fig3. In situ liquid crystal gel before(A) and after steering(B)

\subsubsection{Determination of the gelling capacity}

It was observed that the best prescription P1 showed the "+ +" grade of gelation ability, which is reported to be the most satisfactory grade ${ }^{[27]}$. The precursor formulation was gelatinization immediately after exposure to $\mathrm{PN}$ at $37{ }^{\circ} \mathrm{C}$ degrees can remain for a long time.

\subsection{Characterization of preparations}

\subsubsection{PLM}

We put the samples under the PLM for observation.The precursor formulations(F0,F1,F2,F3) showed a black background under PLM, the liquid crystal gel(H0,H1,H2,H3) formed after phase inversion had a clear fan-shaped texture-like optical birefringence structure observed under PLM, and it can be confirmed that it is a hexagonal phase liquid crystal. H0, H1 and $\mathrm{H} 2$ had no drug crystals, while $\mathrm{H} 3$ showed a few crystals (as shown in Fig 4).

F0

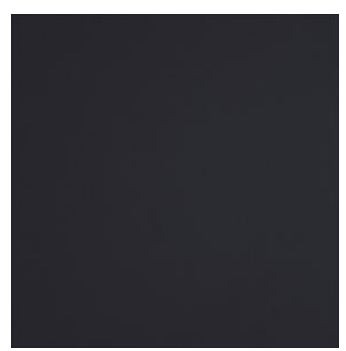

F1

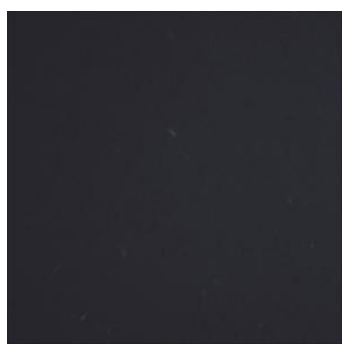

F2

F3 

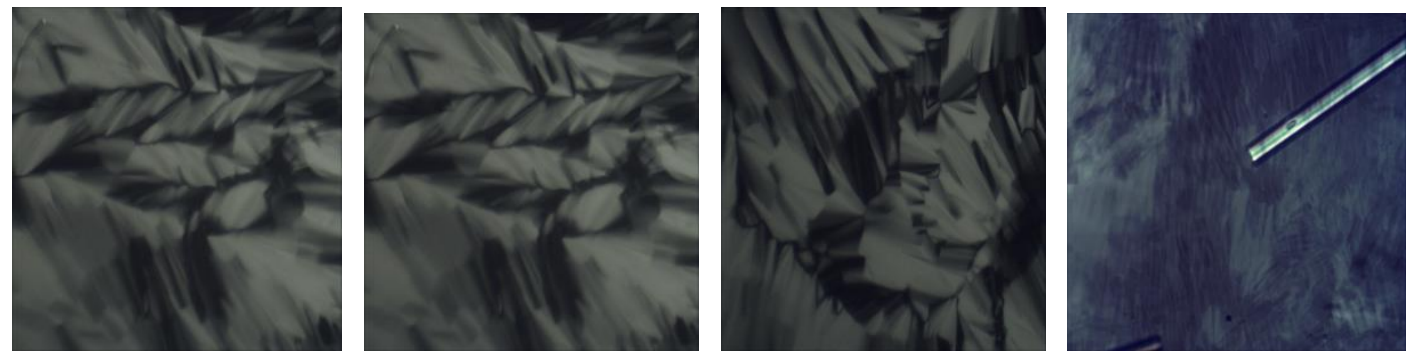

Fig. 4. Observe precursor formulations and liquid crystal gels under PLM

(magnification $\times 100)$

398

399

400

401

402

403

404

405

406

407

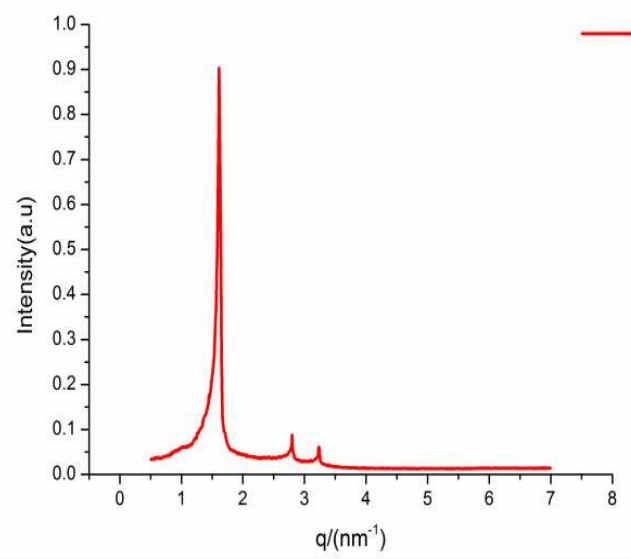

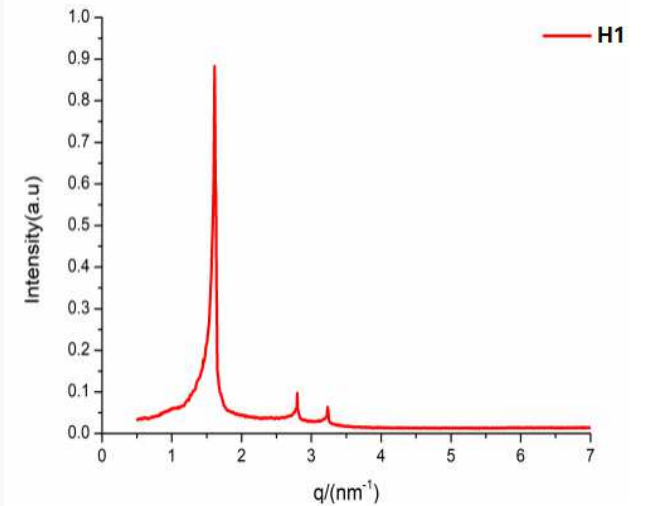

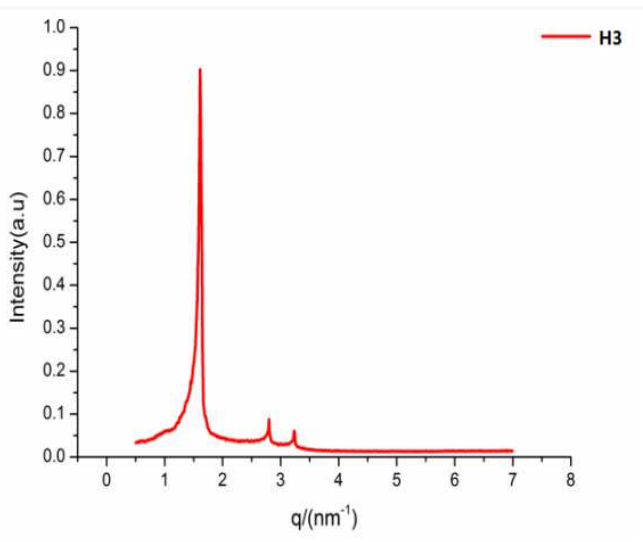


Table 3. Lattice Parameters ( $\alpha$ ) and Layer Spacing (d) of liquid crystal gel

\begin{tabular}{|c|c|c|c|c|}
\hline Formulations & Space group & Bragg peaks & $\alpha\left(\AA^{*}\right)$ & $\mathrm{d}(\AA)$ \\
\hline $\mathrm{H} 0$ & $\mathrm{H}_{\mathrm{II}}$ & $1: \sqrt{ } 2: \sqrt{ } 3$ & 47.95 & 41.52 \\
\hline $\mathrm{H} 1$ & $\mathrm{H}_{\mathrm{II}}$ & $1: \sqrt{ } 2: \sqrt{ } 3$ & 48.16 & 41.71 \\
\hline $\mathrm{H} 2$ & $\mathrm{H}_{\mathrm{II}}$ & $1: \sqrt{ } 2: \sqrt{ } 3$ & 48.58 & 42.07 \\
\hline $\mathrm{H} 3$ & $\mathrm{H}_{\mathrm{II}}$ & $1: \sqrt{ } 2: \sqrt{ } 3$ & 47.32 & 40.98 \\
\hline
\end{tabular}

* $1 \AA=10^{-10} \mathrm{~m}$

Distinctly, the content of PN may affect the internal symmetry of gels mesophases due to the alterations of the lattice parameter. When PN was added to the formulation, the lattice parameter changed from $47.95 \AA$ to $48.58 \AA$. These results could prove that the hydrophilic PN were mainly contained in the water channel, and minimally contained in the lipid-water interface. According to literature research, this may be due to the partial hydration of the polar head group and water in the lyotropic liquid crystal system, which leads to the expansion of the hydrophilic head group of $\mathrm{PT}^{[39]}$. However, the decrease in the value of $\alpha$ in $\mathrm{H} 3$ may be due to the dehydration of the polar head group of PT. When the hydrophilic PN binds more water molecules, it will cause the hydrogen bond between the PT head group and water to decrease, that is, the effective size of the PT head group decreases and the $\alpha$ value decreases ${ }^{[40]}$.

\subsubsection{DSC}

As shown in Fig.7, the thermograms showed a sharp endothermic peak at $190{ }^{\circ} \mathrm{C}$, which corresponded to the melting point of PN in the crystal form. However, the endothermic peaks of PN completely disappeared in the thermograms of PX (Physical mixture) and H3. At the same time, it can be seen from the figure that the melting point of $\mathrm{H} 3$ was $165^{\circ} \mathrm{C}$.

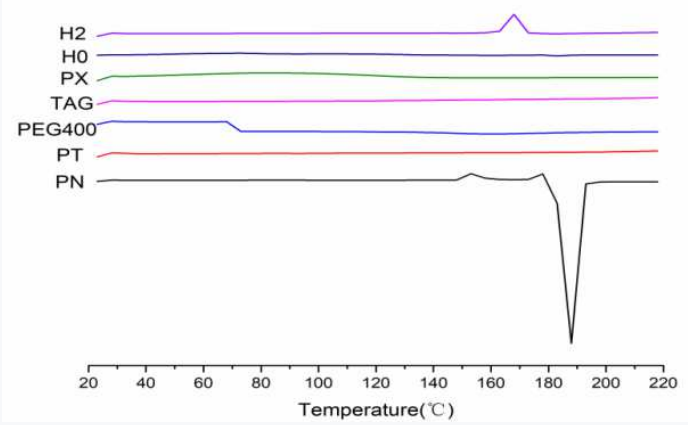

Fig.6. DSC thermograms 


\subsubsection{Rheological characterzation}

It could be seen from the rheological curve of Figure 7(A) that as the shear rate increases, the viscosity of the precursor formulation gradually stabilizes. Obviously, the viscosity of F2 is lower than F0, so the drug can improve the fluidity of the liquid crystal gel precursor formulation ${ }^{[41]}$. When the formulation inverts, the viscosity of the system increases significantly. The results showed that the liquid crystal gels had pseudoplastic flow characteristics (shear thinning system) Viscosity increased at low shear rate and decreased at high shear rate. One of the advantages of shear thinning agents is that they have high viscosity during eye opening and stabilize tear film. When the blink occurs, the gel becomes thinner to prevent the irritation produced by the high viscosity Newtonian fluid ${ }^{[42]}$, so that the preparation is well distributed on the surface of the eye.

Fig.7 (B) showed the relationship between the composite viscosity and the angular frequency. The complex viscosity of the precursor formulations did not change significantly with the angular frequency, but the complex viscosity of the liquid crystal gel decreases with the increase of the angular frequency. These situations were conducive to the uniform dispersion of the gels in the eye.

The experiment adopts the oscillation-frequence test mode. As exhibited in Fig.7 (C), the precursor preparations (F2 and F0) exhibits G" (Viscous modulus) $>\mathrm{G}^{\prime}$ (Elastic Modulus), and the viscous modulus was dominant, showing liquid-like behavior. At the same time, the liquid-like behavior of the precursor formulations were more conducive to the delivery of ophthalmic drugs. When the precursor formulations were transformed into a hexagonal liquid crystal gel, the elastic modulus $G^{\prime}$ and the viscosity modulus $\mathrm{G}$ " are much higher than the formulations before the phase change. Furthermore, after phase inversion to form the liquid crystal gels, $G^{\prime}$ increases with increasing frequency, while $\mathrm{G}^{\prime \prime}$ first rises and then decreases with increasing frequency. However, in the entire scanning process, the values before and after G'" did not change significantly. In the frequency range of $0.01-0.05 \mathrm{~Hz}$, the viscosity modulus was dominant $\left(\mathrm{G}^{\prime}>\mathrm{G}^{\prime}\right)$, but in the frequency range of $0.05-10 \mathrm{~Hz}$, the elastic modulus was dominant $\left(G^{\prime}>G^{\prime}\right)$, It could be concluded that the external frequency changes 
will affect the viscoelasticity of the liquid crystal gel, and we can infer that the liquid crystal gel had less irritation to the eyes at low frequencies ${ }^{[43]}$. It could be concluded that the external frequency changes would affect the viscoelasticity of the liquid crystal gel, and we could deduce that the liquid crystal gel had less irritation to the eyes at low frequencies. At high frequencies, the preparations could resist the damage of high frequency blinking shear force and had good stability. Therefore, the "liquid" behavior of the precursor formulations were more conducive to the delivery of ophthalmic drugs. Besides, the gel-like behavior of liquid crystal gels could make the drug release slowly.

Fig.7 (D) described the effect of temperature on the formulations. Whether it was a precursor preparation or a liquid crystal gel, its viscosity would not change significantly with the increase of temperature, Besides, $\mathrm{H} 5$ was more stable than $\mathrm{H} 0$, indicating that PN could enhance the stability of the internal structure of liquid crystal gel and was suitable for ophthalmology medicine.

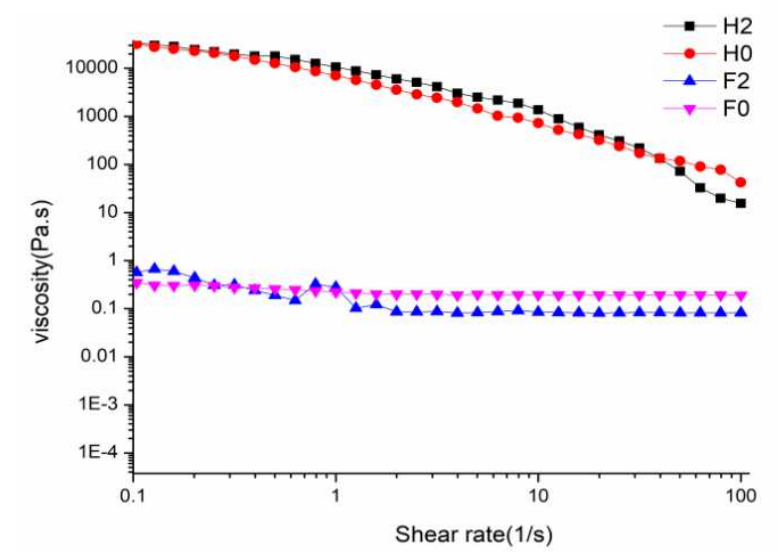

A

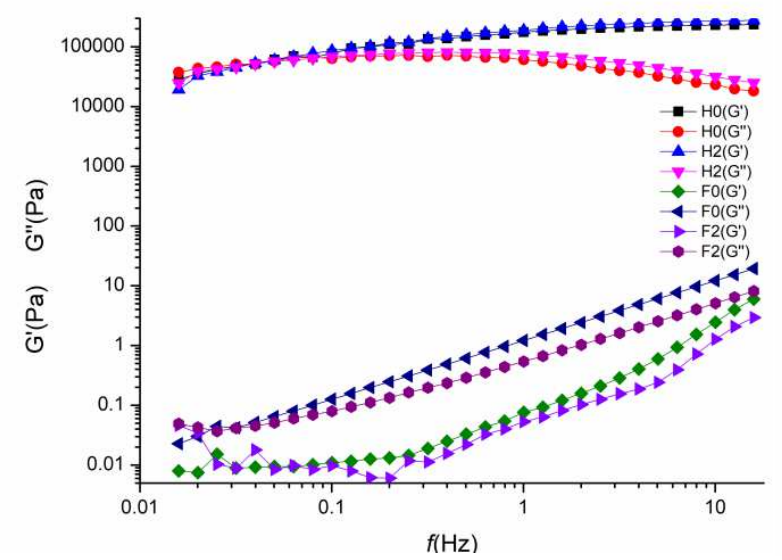

C

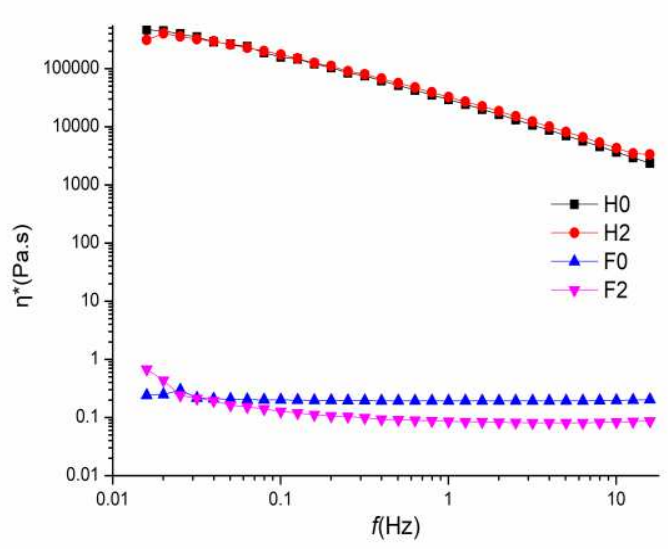

B

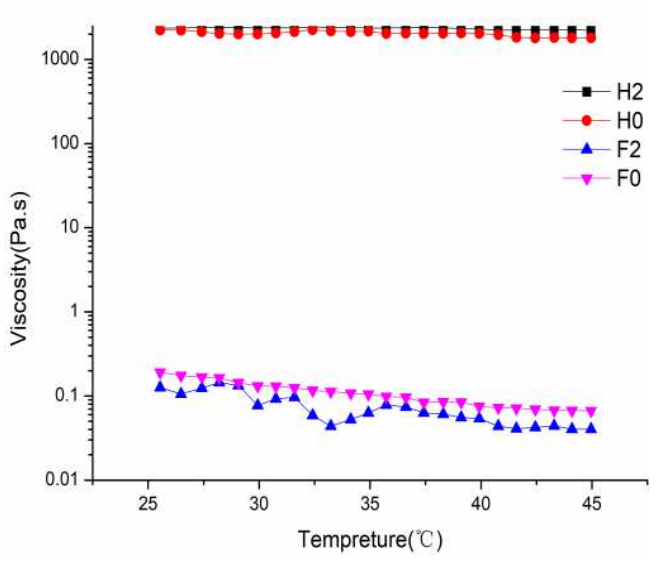

D 
493 Fig.7 (A) Apparent shear viscosity as function shear rate of precursor preparations(F0 494 and $\mathrm{F} 2$ ) and $\mathrm{H}_{\mathrm{II}}$ gels ( $\mathrm{H} 0$ and $\left.\mathrm{H} 2\right)$. Inset exhibits the viscosity at specific shear rate $495 \quad(0.101 / \mathrm{s})$. (B) Complex viscosity of precursor preparations (F0 and F2) and $\mathrm{H}_{\text {II }}$ gels 496 (H0 and H2) at $37{ }^{\circ} \mathrm{C}$ as a function of the applied angular frequency. (C) Variation of 497 elastic modulus $\mathrm{G}^{\prime}$ and viscous modulus $\mathrm{G}^{\prime \prime}$ as a function of frequency for precursor 498 preparations (F0 and F2) and $\mathrm{H}_{\mathrm{II}}$ gels (H0 and H2). (D) Temperature sweep results 499 500 501

\subsection{In vitro studies}

\section{3 \\ 3.3.1 In vitro release study}

As shown in Fig.8, the Qn of H1, H2, H3 in the previous hour were 43.84\%, reached $98.59 \%$ within two hours. Obviously, the drug release increased by approximately 2-folds from eye drops in comparison to the correponding $\mathrm{H}_{\text {II }}$ gels. It could be speculated that $\mathrm{H}_{\text {II }}$ gels has a good sustained-release effect compared with traditional eye drops.

511 drug release rate is influenced by the lattice parameters and the diameter of water 512 cylinders. Therefore, we can deduce that although H3 contains the largest drug 513 loading, its lattice parameters and water channel diameters are smaller than those of $514 \mathrm{H} 1$ and H2, which was supported by the outcomes of SAXS. The release trends of the 515 three drug loads were similar. However, in terms of cumulative release, H2 was higher than $\mathrm{H} 1$ and $\mathrm{H} 3$. According to the above results, $\mathrm{H} 2$ has the best drug release 517 effect, and we will choose $\mathrm{H} 2$ for the following experiments. 


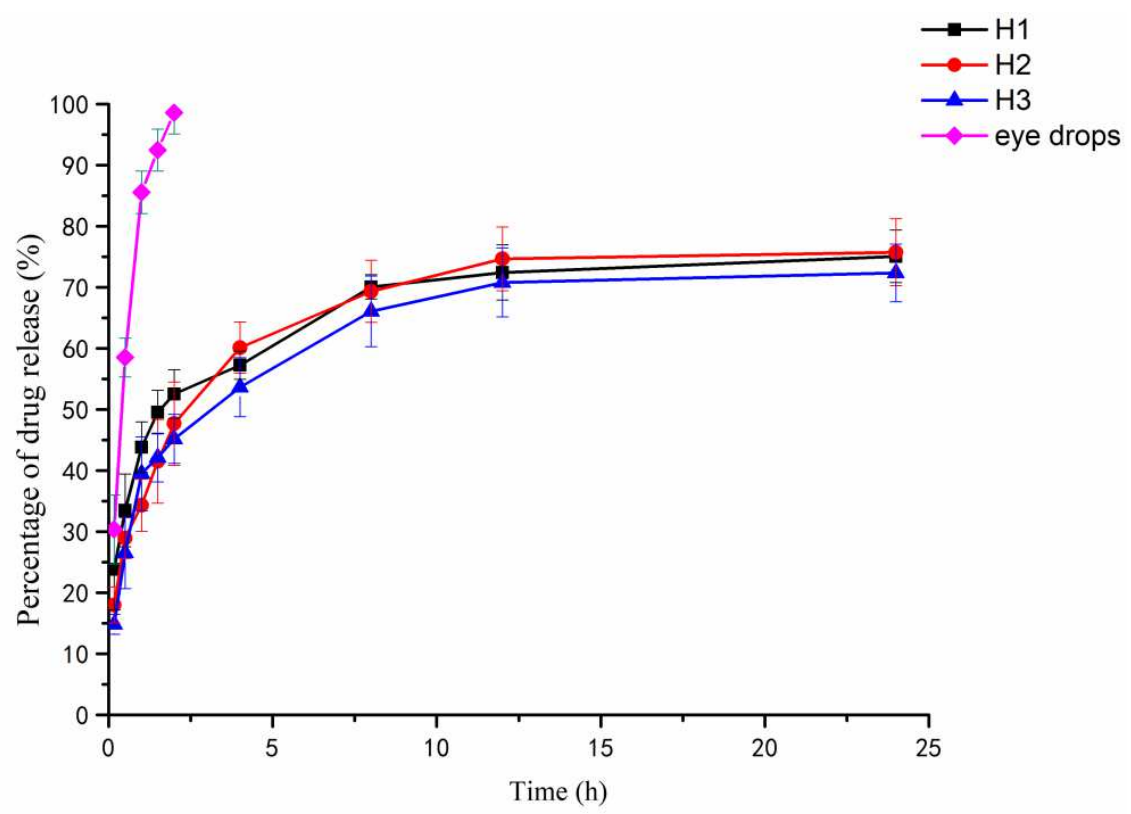

518

519

520

521

522

523 larger than other models, so it proved to be diffusion controlled release ${ }^{[44]}$. The

524 Ritger-Peppas model was used to evaluate the drug release mechanism. The ' $n$ ' of the

525 three preparations was less than 0.45 , so the drug was controlled by Fickian

526 diffusion $^{[45]}$.

527

528

529

\begin{tabular}{|c|c|c|c|c|c|}
\hline \multirow{2}{*}{ Formulations } & \multirow{2}{*}{$\begin{array}{c}\text { Zero-order } \\
\mathbf{R}^{2}\end{array}$} & \multirow{2}{*}{$\begin{array}{c}\text { First-order } \\
\mathbf{R}^{2}\end{array}$} & \multirow{2}{*}{$\begin{array}{c}\text { Higuchi } \\
\text { model } \\
\mathbf{R}^{2}\end{array}$} & \multicolumn{2}{|c|}{ Ritger-Peppas } \\
\hline & & & & $\mathbf{R}^{2}$ & $\mathbf{n}$ \\
\hline $\mathrm{H} 1$ & 0.6941 & 0.7395 & 0.8432 & 0.9576 & 0.2366 \\
\hline $\mathrm{H} 2$ & 0.6331 & 0.7319 & 0.8465 & 0.9583 & 0.3024 \\
\hline H3 & 0.6324 & 0.7373 & 0.8420 & 0.9313 & 0.3151 \\
\hline Eye drops & 0.3567 & 0.4330 & 0.5247 & 0.8041 & 0.0243 \\
\hline
\end{tabular}




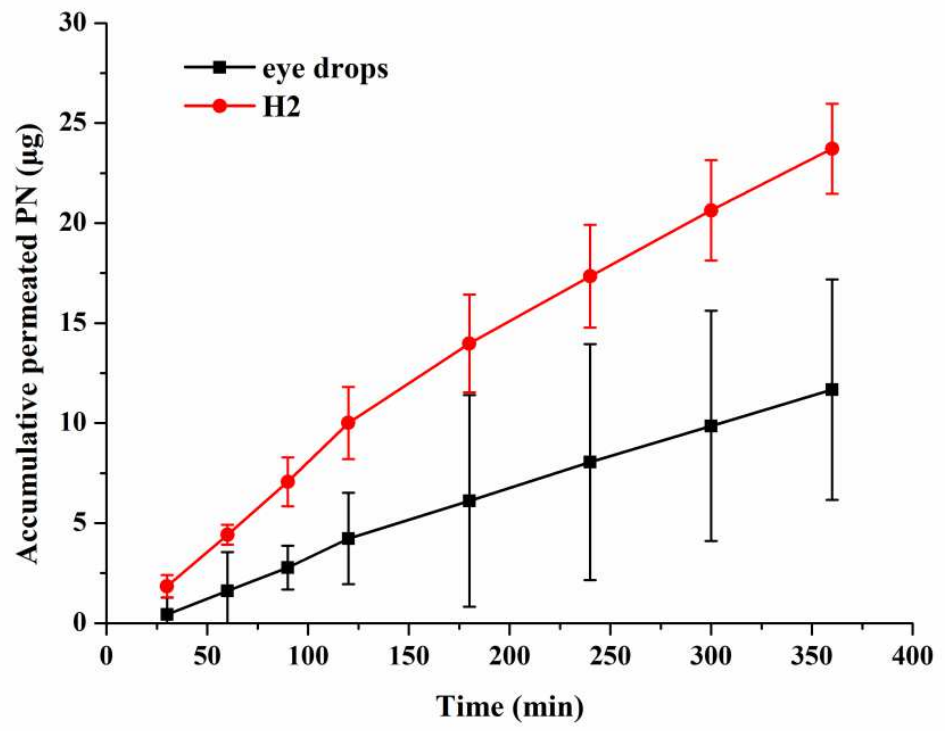

Fig.9 In vitro corneal permeability curve of $\mathrm{H}_{\text {II }}$ gels and $\mathrm{PN}$ eye drops

Table 5. In vitro corneal osmotic parameters of in situ liquid crystal and PN eye drops All the data are representative $(\bar{x} \pm \mathrm{SD}, \mathrm{n}=3)$

\begin{tabular}{ccc}
\hline Formulations & $\begin{array}{c}\mathrm{J}_{\mathrm{ss}} \times 10^{2} / \\
\left(\mu \mathrm{g} \cdot \mathrm{s}^{-1} \mathrm{~cm}^{-2}\right)\end{array}$ & $\begin{array}{c}\mathrm{P}_{\mathrm{app}} \times 10^{6} / \\
\left(\mathrm{cm} \cdot \mathrm{s}^{-1}\right)\end{array}$ \\
\hline $\mathrm{H} 2$ & $3.5 \pm 0.021 *$ & $0.35 \pm 0.18 *$ \\
Eye drops & $0.055 \pm 0.018$ & $0.11 \pm 0.15$ \\
\hline
\end{tabular}

$* \mathrm{P}<0.05$, which is statistically different from eye drops.

Fig. 10 showed the corneal penetration curve of $\mathrm{H}_{\text {II }}$ gel and eye drops. Obviously, the corneal permeability of the liquid crystal gel group was higher than that eye drops group. It could be seen from Table 5. that the apparent permeability coefficients of H2 and eye drops were $0.35 \times 10^{6}$ and $0.11 \times 10^{6} \mathrm{~cm} / \mathrm{s}$, respectively. Compared with commercially available drugs, the apparent permeability coefficient of H2 was 3.19 times that of eye drops, indicating that the rabbit cornea penetrates much more PN than eye drops.

First of all, we speculate that this difference may be due to the good biocompatibility of biolipid carriers and corneal epithelial cells, which leads to 
554 increased solubility of the drug, which makes it easier to penetrate the corneal

555 barrier $^{[45]}$. Furthermore, many literatures demonstrated that PT can promote the

556 transdermal absorption of hydrophilic drugs ${ }^{[46]}$. Therefore, it is inferred that PT itself

557 has permeability promoting properties and can enhance the permeability of the

558 corneal epithelium ${ }^{[47,48]}$.

$559 \quad$ 3.3.3 Corneal hydration level

560 By calculating the HL value to assess the tissue damage to the cornea. The HL

561 value of a healthy cornea is between $76 \sim 80 \%$, and the corneal hydration value

562 exceeds $83 \%$, indicating a certain degree of corneal damage. The HL value of the eye

563 drops was higher than $80 \%$, while the HL value of the $\mathrm{H}_{\text {II }}$ gels preparation was $76.91 \pm$

$5640.43 \%$. The results showed that the $\mathrm{H}_{\text {II }}$ gels would not produce obvious corneal

565 irritation and damage.

566

567

4.4 In vivo study

568

4.4.1 Ocular irritation test

569

As shown in Table 6, after a single administration, the in situ liquid crystal and 570 eye drops had little effect on the cornea and iris, the cornea was not turbid, and the conjunctiva was free of redness, congestion, swelling and other irritation. After

572 repeated administration, the liquid crystal gel would be located in the subconjunctival 573 sac of the rabbit's eye. The conjunctiva may be slightly reddened due to blinking 574 reaction, but the score was lower than 3 . The comprehensive results showed that both 575 in situ liquid crystal and PN eye drops had less irritating to rabbit eyes.

576

577

Table 6. Evaluation results of ocular irritation in single and multiple doses of improved Draize test $(n=6)$

579

\begin{tabular}{ccccccc}
\hline \multirow{2}{*}{ Location } & \multicolumn{2}{c}{ Physiological saline } & \multicolumn{2}{c}{ Eye drops } & \multicolumn{2}{c}{ F5 } \\
\cline { 2 - 7 } & Single & Long-term & Single & Long-term & Single & Long-term \\
\hline Cornea & 0 & 0 & 0 & 0 & 0 & 0 \\
Iris & 0 & 0 & 0 & 0 & 0 & 0 \\
\hline
\end{tabular}




\begin{tabular}{ccccccc}
\hline $\begin{array}{c}\text { Conjunctival } \\
\text { congestion }\end{array}$ & 0 & 0 & 0.2 & 0.4 & 0 & 0.4 \\
$\begin{array}{c}\text { Conjunctival } \\
\text { edema }\end{array}$ & 0 & 0.5 & 0.1 & 0.2 & 0.3 & 1.2 \\
$\begin{array}{c}\text { Secretions } \\
\text { Sum score }\end{array}$ & 0 & 0 & 0.1 & 0 & 0 & 0 \\
\hline
\end{tabular}

580

4.4.2 Pre-Corneal Residence Time Analysis

582

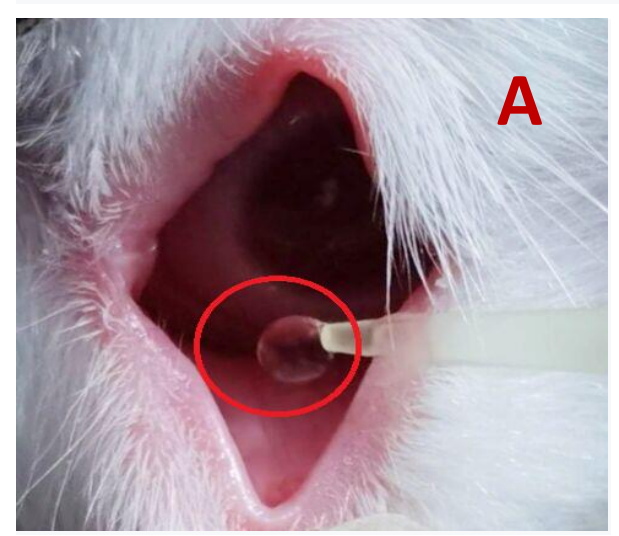

583
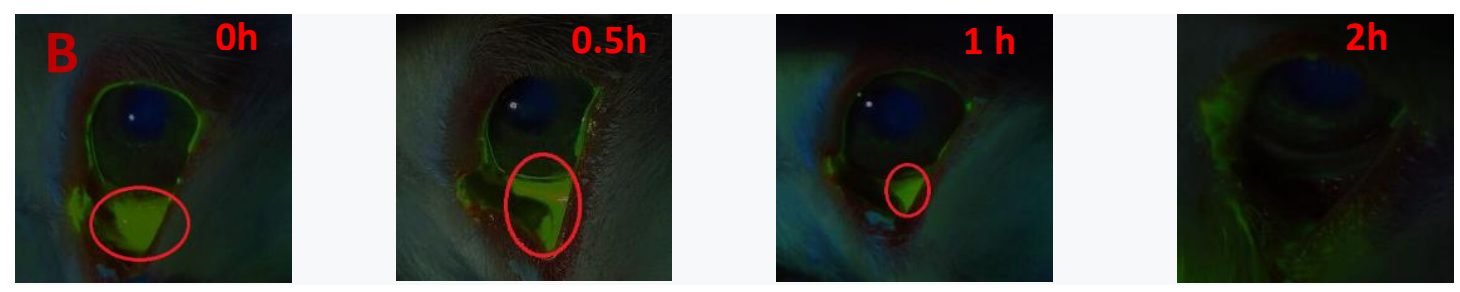

585

586
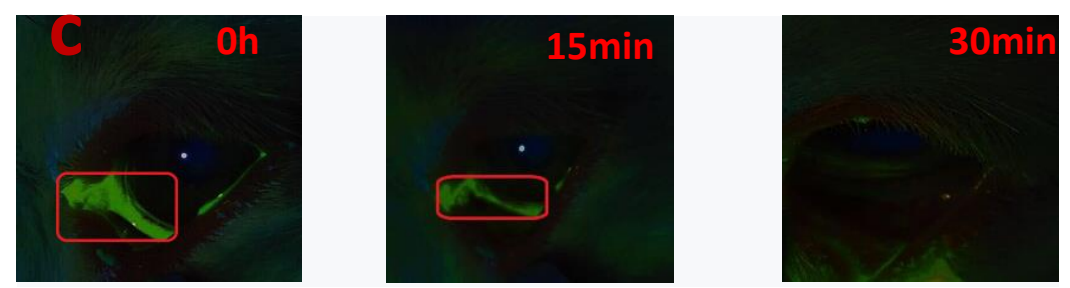

Fig. 10. Pre-corneal distribution of administration of the formulations at specified time points. (A) Anterior surface of eyeball, (B) $\mathrm{H}_{\text {II }}$ gel, (C) eye drops.

As displayed in Fig. 10, the $\mathrm{H}_{\text {II }}$ gel could observe a stronger fluorescence 591 intensity. Compared with eye drops, the fluorescent signal of the $H_{\text {II }}$ gel was distributed in the subconjunctival capsule for to $2 \mathrm{~h}$. Obviously, the rabbit eyes coated 
593 with eye drops did not show strong fluorescence intensity and were quickly cleared 594 after $30 \mathrm{~min}$, which indicates that the retention time of eye drops in the eye was very 595 short. This showed that compared with traditional eye drops, $\mathrm{H}_{\text {II }}$ gel could significantly

596

597

598

599

600

601

602

603

604

605

606

607

608

609

610

611

612

613

614

615

616

617

618

619

620

621

increase the contact time of the drug in front of the cornea. We speculated that the good bioadhesion of PT leaded to relatively strong fluorescence intensity and slow clearance. Secondly, PT may interacted with the mucin in the corneal epithelium, thereby shortening the residence time of the drug on the corneal surface ${ }^{[49]}$, or PT may have a certain degree of biodegradability in the body, causing the fluorescence intensity to gradually decrease over time. Generally, $\mathrm{H}_{\text {II }}$ gel has good bioadhesion and would not reduce the residence time due to blinking, which has been verified by previous rheological results.

5 Conclusion

In summary, compared with eye drops, the liquid crystal gel precursor formulation significantly improves the bioavailability and biocompatibility of the drug. The in vitro release test results show that the liquid crystal gel had a better sustained-release effect, avoiding the shortcomings of repeated administration. In vitro corneal penetration experiments showed that liquid crystal gel could enhance corneal penetration. In addition, the corneal hydration level and Draize test proved that the preparation was less irritating to the eyes and was suitable for ocular administration. The analysis of the pre-corneal residence time proved that the preparation has good bioadhesion. In short, the liquid crystal gel precursor formulation had good fluidity and convenient administration. It had good bioadhesion, sustained release, good corneal permeability and low irritation. The research results provided a new way and method for the clinical treatment of glaucoma or other eye diseases, and provide a theoretical basis and reference for other topical drug research.

\section{Availability of data and material}

The datasets used or analysed during the current study are available from the corresponding author on reasonable request.

\section{Declaration of interest}


The authors declare that there is no conflict of interest.

624

625

626

627

628

629

630

631

632

633

634

635

636

637

638

639

640

641

642

643

644

645

646

647

648

649

650

651

652

653

654

655

656

657

\section{Funding Information}

The authors gratefully acknowledge support from the National Natural Science Foundation of China (No.81803831), Key University natural science research project of Anhui province (KJ2018A0301). and Anhui Provincial Talents Project for youth in Universities (No.gxyq2018025).

\section{Acknowledgements}

The content is solely the responsibility of the authors and does not necessarily represent the official views of Anhui University of Chinese Medicine.

\section{Conflict of interest}

The authors declared that they have no conflicts of interest to this work. We declare that we do not have any commercial or associative interest that represents a conflict of interest in connection with the work submitted.

\section{References}

[1] Li Q, Wang J, Shahani S, et al. Biodegradable and photocrosslinkable polyphosphoester hydrogel. Biomaterials. 2006;27(7):1027-1034.

[2] Dong Y, Hassan WU, Kennedy R, et al. Performance of an in situ formed bioactive hydrogel dressing from a PEG-based hyperbranched multifunctional copolymer. Acta Biomater. 2014;10(5):2076-2085.

[3] Nirmal H, Bakliwal S, Pawar S. In-situ gel: new trends in controlled and sustained drug delivery system. Int J PharTech Res. 2010;2(2):1398-1408.

[4] Fakhari A, Corcoran M, Schwarz A. Thermogelling properties of purified poloxamer 407. Heliyon. 2017;3(8):e00390. Published 2017 Aug 30.

[5] Pereira G, Dimer F, Guterres S. Formulation and characterization of Poloxamer 407®: thermoreversisble gel containing polymeric microparticles and hyaluronic acid. Quim Nova. 2013;36(8):1121-5.

[6] Wen-Di M, Wang C, Nie S, Pan W. Pluronuc F127-g-poly (acrylic acid) copolymers as in situ gelling vehicle for ophthalmic drug delivery system. Int J Pharm. 2007;350:247-56.

[7] Edsman K, Carfors J, Petersson R. Rheological evaluation of polxamer as an in situ gel for ophthalmic use. Eur J Pharm Sci. 1998;6:105-12. 
[8] Phaechamud T, Mahadlek J. Solvent exchange-induced in situ forming gel comprising ethyl cellulose-antimicrobial drugs. Int J Pharm. 2015;494(1):381-392.

[9] Narurkar MM, Mitra AK. Prodrugs of 5-iodo-2'-deoxyuridine for enhanced ocular transport. Pharm Res. 1989;6(10):887-891.

[10] Srividya B, Cardoza R, Amin P . Sustained ophthalmic delivery of ofloxacin from a $\mathrm{pH}$ triggered in situ gelling system. J Control Release. 2001;73:205-211.

[11] Wu H, Liu Z, Peng J, Li L, Li N, Li J, et al. Design and evaluation of baicalin-containing in situ pH-triggered gelling system for sustained ophthalmic drug delivery. Int J Pharm.2011;410:31-40.

[12] Jain SP, Shah SP, Rajadhyaksha NS, Singh P S PP, Amin PD. In situ ophthalmic gel of ciprofloxacin hydrochloride for once a day sustained delivery. Drug Dev Ind Pharm. 2008;34(4):445-452

[13] Fan J, Liu F, Wang Z. Shear rheology and in-vitro release kinetic study of apigenin from lyotropic liquid crystal. Int J Pharm. 2016;497(1-2):248-254.

[14] Mei L, Xie Y, Huang Y, et al. Injectable in situ forming gel based on lyotropic liquid crystal for persistent postoperative analgesia. Acta Biomater. 2018;67:99-110.

[15] Quigley HA, Broman AT. The number of people with glaucoma worldwide in 2010 and 2020. Br J Ophthalmol. 2006;90(3):262-267.

[16] Wei J, He HL, Zheng CL, Zhu JB. Yao Xue Xue Bao. 2011;46(8):990-996.

[17] Nair KL, Vidyanand S, James J, Kumar,et al. Pilocarpine-loaded poly (dllactic-co-glycolic acid) nanoparticles as potential candidates for controlled drug delivery with enhanced ocular pharmacological response. J.Appl. Polym. Sci. 2012;124 (3), 2030-2036.

[18] Aktaş Y, Unlü N, Orhan M, Irkeç M, Hincal AA. Influence of hydroxypropyl beta-cyclodextrin on the corneal permeation of pilocarpine. Drug Dev Ind Pharm. 2003;29(2):223-230.

[19] Zimmerman TJ. Pilocarpine. Ophthalmology. 1981;88(1):85-88.

[20] Kao HJ, Lin HR, Lo YL, Yu SP. Characterization of pilocarpine-loaded chitosan/Carbopol nanoparticles. J Pharm Pharmacol. 2006;58(2):179-186.

[21] Wang X, Zhang Y, Huang J, et al. Self-assembled hexagonal liquid crystalline gels as novel ocular formulation with enhanced topical delivery of pilocarpine nitrate. Int $\mathrm{J}$ Pharm. 2019;562:31-41.

[22] Coscia BJ, Yelk J, Glaser MA, Gin DL, Feng X, Shirts MR. Understanding the Nanoscale Structure of Inverted Hexagonal Phase Lyotropic Liquid Crystal Polymer Membranes. J Phys Chem B. 2019;123(1):289-309.

[23] Xingqi W, Yong Z, Xing L, et al. Cubic and hexagonal liquid crystal gels for ocular delivery with enhanced effect of pilocarpine nitrate on anti-glaucoma treatment. Drug Deliv. 2019;26(1):952-964.

[24] Zabara A, Mezzenga R. Controlling molecular transport and sustained drug release in lipid-based liquid crystalline mesophases. J Control Release. 2014;188:31-43

[25] Chen YL, Gui SY, Liang X. Preparation and evaluation of intraarticular injectable sinomenine hydrochloride-loaded in-situ liquid crystals. Acta Pharm Sin. 2016;51:132-139

[26] Qi, H., Chen, W., Huang, C., Li, L., Chen, C., Li, W., Wu, C., 2007. Development of apoloxamer analogs/carbopol-based in situ gelling and mucoadhesive ophthalmic delivery system for puerarin. Int. J. Pharmaceut. 337 (1), 178-187. 
[27] Jie H, Liu L, Shuangying G, et al. A Novel Phytantriol-Based In Situ Liquid Crystal Gel for Vaginal Delivery. AAPS PharmSciTech. 2019;20(5):185.

[28] Milak S, Zimmer A. Glycerol monooleate liquid crystalline phases used in drug delivery systems. Int J Pharm. 2015;478(2):569-587.

[29] Chen Y, Liang X, Ma P, et al. Phytantriol-based in situ liquid crystals with long-term release for intra-articular administration. AAPS PharmSciTech. 2015;16(4):846-854.

[30] Báez-Santos YM, Otte A, Mun EA, et al. Formulation and characterization of a liquid crystalline hexagonal mesophase region of phosphatidylcholine, sorbitan monooleate, and tocopherol acetate for sustained delivery of leuprolide acetate. Int J Pharm. 2016;514(1):314-321.

[31] Ye J, Wang Q, Zhou X, Zhang N. Injectable actarit-loaded solid lipid nanoparticles as passive targeting therapeutic agents for rheumatoid arthritis. Int J Pharm. 2008;352(1-2):273-279.

[32] Wang X, Zhang Y, Huang J, et al. A Novel Phytantriol-Based Lyotropic Liquid Crystalline Gel for Efficient Ophthalmic Delivery of Pilocarpine Nitrate. AAPS PharmSciTech. 2019;20(1):32. Published 2019 Jan 2.

[33] Moustafa MA, El-Refaie WM, Elnaggar YSR, Abdallah OY. Gel in core carbosomes as novel ophthalmic vehicles with enhanced corneal permeation and residence. Int $\mathrm{J}$ Pharm. 2018;546(1-2):166-175.

[34] Yu S, Wang QM, Wang X, et al. Liposome incorporated ion sensitive in situ gels for opthalmic delivery of timolol maleate. Int J Pharm. 2015;480(1-2):128-136.

[35] Bhatta RS, Chandasana H, Chhonker YS, et al. Mucoadhesive nanoparticles for prolonged ocular delivery of natamycin: In vitro and pharmacokinetics studies. Int $\mathrm{J}$ Pharm. 2012;432(1-2):105-112.

[36] Fiscella R G . Ophthalmic Drug Formulations - ScienceDirect[J]. Clinical Ocular Pharmacology (Fifth Edition), 2008:17-37.

[37] Phan S, Fong WK, Kirby N, Hanley T, Boyd BJ. Evaluating the link between self-assembled mesophase structure and drug release. Int J Pharm. 2011;421(1):176-182.

[38] Fong WK, Hanley T, Boyd BJ. Stimuli responsive liquid crystals provide 'on-demand' drug delivery in vitro and in vivo. $J$ Control Release. 2009;135(3):218-226.

[39] Amar-Yuli I, Garti N. Transitions induced by solubilized fat into reverse hexagonal mesophases. Colloids Surf B Biointerfaces. 2005;43(2):72-82.

[40] Chang CM, Bodmeier R. Swelling of and drug release from monoglyceride-based drug delivery systems. J Pharm Sci. 1997;86(6):747-752.

[41] Wang B, Huang Y, Huang Z, et al. Self-assembling in situ gel based on lyotropic liquid crystals containing VEGF for tissue regeneration. Acta Biomater. 2019;99:84-99.

[42] Mezzenga R, Meyer C, Servais C, Romoscanu AI, Sagalowicz L, Hayward RC. Shear rheology of lyotropic liquid crystals: a case study. Langmuir. 2005;21(8):3322-3333.

[43] Wan J, Wang SM, Gui ZP, et al. Phytantriol-based lyotropic liquid crystal as a transdermal delivery system. Eur J Pharm Sci. 2018;125:93-101.

[44] Elgindy NA, Mehanna MM, Mohyeldin SM. Self-assembled nano-architecture liquid crystalline particles as a promising carrier for progesterone transdermal delivery. Int $\mathrm{J}$ Pharm. 2016;501(1-2):167-179.

[45] Unagolla JM, Jayasuriya AC. Drug transport mechanisms and in vitro release kinetics of vancomycin encapsulated chitosan-alginate polyelectrolyte microparticles as a controlled drug delivery system. Eur J Pharm Sci. 2018;114:199-209. 
[46] Chu XQ, Huang J, Li ZG, et al. On the Structure and Transdermal Profile of Liquid Crystals

747 Based on Phytantriol. Curr Drug Deliv. 2018;15(10):1439-1448.

748 [47] Agibayeva LE, Kaldybekov DB, Porfiryeva NN, et al. Gellan gum and its methacrylated

749 derivatives as in situ gelling mucoadhesive formulations of pilocarpine: In vitro and in vivo

750 studies. Int J Pharm. 2020;577:119093.

751 [48] Destruel PL, Zeng N, Seguin J, et al. Novel in situ gelling ophthalmic drug delivery system

752 based on gellan gum and hydroxyethylcellulose: Innovative rheological characterization, in vitro

753 and in vivo evidence of a sustained precorneal retention time. Int J Pharm. 2020;574:118734.

754 [49] Zhao R, Li J, Wang J, Yin Z, Zhu Y, Liu W. Development of Timolol-Loaded Galactosylated

755 Chitosan Nanoparticles and Evaluation of Their Potential for Ocular Drug Delivery. AAPS

756 PharmSciTech. 2017;18(4):997-1008.

757 